\title{
ESCANEAMENTO A LASER EM TRÊS DIMENSÕES E MÉTODOS DE LEVANTAMENTO INTEGRADOS: REFLEXÃO CRÍTICA A PARTIR DA COOPERAÇÃO FAU USP-DIAPREM
}

\section{LASER SCANNING IN THREE DIMENSIONS AND INTEGRATED SURVEY METHODS: CRITICAL REFLECTION FROM THE FAUUSP-DIAPREM COOPERATION}

\author{
Beatriz Mugayar Kühl ${ }^{1}$, Renata Cima Campiotto ${ }^{1}$, Marcello Balzani ${ }^{2}$, Federica Maietti ${ }^{2}$ \\ Luca Rossato ${ }^{2}$, Fabiana Raco ${ }^{2}$
}

\section{RESUMO:}

Este artigo aborda métodos integrados de diagnóstico de bens arquitetônicos e tem por objetivo evidenciar as características e potencialidades do escaneamento a laser em três dimensões, a partir da experiência de cooperação científica entre a Faculdade de Arquitetura e Urbanismo da Universidade de São Paulo (FAU USP) e o laboratório de pesquisa da Università degli Studi di Ferrara (Unife) - Development of Integrated Automatic Procedures for Restoration of Monuments (DIAPReM). A análise é centrada nas experiências conjuntas entre as instituições, voltadas ao Edifício Vilanova Artigas, sede da FAU USP, e ao Museu do Ipiranga da USP. Para estruturar as questões, são inicialmente apresentadas as características da colaboração entre as instituições para, depois, analisar as características dos trabalhos feitos no Edifício Vilanova Artigas e no Museu do Ipiranga. Em seguida, são examinadas as particularidades do método e a discussão sobre suas potencialidades durante os ciclos de formação de estudantes da FAU USP; depois, são apresentadas considerações sobre o escaneamento a laser e seu papel no diagnóstico integrado. $O$ texto é encerrado com reflexões sobre o método e suas potencialidades, evidenciando a necessidade de uma abordagem crítica.

PALAVRAS-CHAVE: Patrimônio arquitetônico; Levantamento integrado; Escaneamento a laser 3D.

\begin{abstract}
:
This article discusses integrated survey methods for architectural assets. Aims at highlighting the characteristics and potential of 3D laser scanning. The text is based on the experience of the scientific cooperation between Faculdade de Arquitetura e Urbanismo of the Universidade de São Paulo (FAU USP) and the research laboratory of the Università degli Studi di Ferrara (Unife) - Development of Integrated Automatic Procedures for Restoration of Monuments (DIAPReM). The analysis is centered on the joint experiences between the institutions on the Vilanova Artigas Building, headquarters of FAU USP, and Ipiranga Museum USP. To structure the text, the characteristics of the collaboration between the institutions are initially presented, followed by the analysis of the works done in the Vilanova Artigas Building and in the Ipiranga Museum. The particularities of the method and the discussion of its potentialities during the training cycles of FAU USP students are then examined, followed by considerations about laser scanning and its role in the integrated survey methods. The text ends with reflections on the method and its potential, emphasizing the need for a critical approach.
\end{abstract}

KEYWORDS: Architectural heritage; Integrated survey methods; 3D laser scanning.
${ }^{1}$ Faculdade de Arquitetura e Urbanismo da Universidade de São Paulo

2 Dipartimento di Architettura dell'Università degli Studi di Ferrara

Fonte de Financiamento: CNPq (Bolsa de Produtividade em Pesquisa), FAPESP (Bolsa de Doutorado Direto $-n^{0}$ 2019/10406-0), The Getty Foundation (Programa Keeping It Modern), Fundação de Amparo à Universidade de São Paulo.

Conflito de Interesse: Declara não haver.

Ética em Pesquisa: Declara não haver necessidade.

Submetido em: 03/09/2020 Aceito em: 11/02/2021

How to cite this article:

KÜHL, B. M. et. al. Escaneamento a laser em três dimensões e métodos de levantamento integrados: reflexão crítica a partir da cooperação FAU USP-DIAPReM. Gestão \& Tecnologia de Projetos. São Carlos, v16, n3, 2021. https://dx.doi.org/10.11606/gtp.v16Xi3.174457 


\section{INTRODUÇÃO}

Este artigo aborda métodos integrados de diagnóstico de bens arquitetônicos, com o intuito de evidenciar as características do escaneamento a laser em três dimensões, a partir da experiência de cooperação entre a Faculdade de Arquitetura e Urbanismo da Universidade de São Paulo (FAU USP) e o laboratório de pesquisa da Università degli Studi di Ferrara (Unife) Development of Integrated Automatic Procedures for Restoration of Monuments (DIAPReM). As análises são centradas nas experiências conjuntas entre as instituições, que foram voltadas ao Edifício Vilanova Artigas, sede da FAU USP, e ao edifício do Museu do Ipiranga, da USP. Para desenvolver o tema, são inicialmente apresentadas as características da colaboração entre as instituições para, depois, examinar as características dos trabalhos feitos no Edifício Vilanova Artigas e no Museu do Ipiranga. Em seguida são perscrutadas as particularidades do método e discutidas suas potencialidades durante os ciclos de formação de estudantes da FAU USP. Por fim, são apresentadas considerações sobre o escaneamento a laser e seu papel no diagnóstico integrado, com reflexões sobre o método, suas características e potencialidades.

A FAU USP e o DIAPReM desenvolvem projetos de cooperação científica desde 2015, quando foi assinado o acordo entre a USP, por meio do Centro de Preservação Cultural (CPC USP) e o laboratório de Ferrara. A colaboração é voltada para questões relacionadas à preservação de bens culturais e não se resume a mera prestação de serviço para o escaneamento a laser em três dimensões (3D) de edifícios da USP. Pelo contrário, prevê o desenvolvimento de pesquisas conjuntas, examinando questões de método sobre o levantamento diagnóstico integrado - que envolve o escaneamento a laser e outras formas de levantamento e diagnóstico - e abarca a formação de pessoal, intercâmbio de conhecimentos e transferência de tecnologia, realizando, de fato, trabalhos integrados.

O primeiro projeto conjunto foi voltado às empenas do Edifício Vilanova Artigas, sede da FAU USP na Cidade Universitária, contemplado por financiamento da Fundação Getty, através do programa Keeping It Modern, que se estendeu de 2015 a 2017. 0 segundo, foi centrado no edifício do Museu do Ipiranga e realizado entre 2017 e 2018. 0 escaneamento a laser permite obter a geometria do edifício com alto grau de precisão e oferece dados das superfícies que podem ser analisados qualitativamente; possibilita, assim, que a nuvem de pontos seja indagada de diversos modos, como para fins de diagnóstico estrutural, controlando deformações existentes ou para identificar degradações nas superfícies. Os dados também devem ser articulados com pesquisa documental, iconográfica e bibliográfica para a compreensão mais aprofundada da história do edifício e de seus aspectos construtivos, sendo fonte para esclarecimentos historiográficos. Com grande quantidade de informações que podem ser interpretadas do ponto de vista qualitativo-analítico, a nuvem de pontos retrata o edifício num determinado momento, e pode ser usada como base de comparação com outros escaneamentos realizados ao longo do tempo, permitindo controlar, por exemplo, o comportamento de estruturas após obras de estabilização.

Os trabalhos entre as equipes da FAU USP e do DIAPReM buscam também maior conscientização sobre o próprio método do escaneamento a laser, sobre o qual existem equívocos de percepção, tratados adiante. As atividades foram estruturadas em reuniões de trabalho entre os coordenadores das pesquisas, seminários voltados à formação de equipe de estudantes da FAU USP para trabalhar com a nuvem de pontos, diversas apresentações voltadas a público mais amplo, participação em workshops e congressos e divulgação dos resultados desse processo em relatórios científicos (PINHEIRO et al., 2017), artigos em periódicos arbitrados (BALZANI; MAIETTI; KÜHL, 2017; BALZANI et al., 2020) e numerosos eventos científicosi. As discussões das equipes, assim como as apresentações abertas ao público, tiveram por objetivo debater as características metodológicas do levantamento 
arquitetônico integrado e da tecnologia do escaneamento a laser a partir da análise de estudos de caso. Foram apresentados os conceitos básicos da nuvem de pontos, evidenciando os tipos de visualização possíveis (renderings) e como é feita a extração de elaborados gráficos, como plantas, cortes e modelos geométricos eletrônicos. Outra ênfase foi trabalhar com a nuvem de pontos gerada pelo escaneamento de modo integrado a outros métodos para analisar as degradações das superfícies. Pensando em um processo cumulativo e gradual de formação e experiências conjuntas, é importante especificar que a execução do escaneamento e o tratamento dos dados foram feitos pelo DIAPReM. Prevê-se, em uma nova fase, que a equipe da FAU USP possa participar ativamente do escaneamento até ter condições de fazê-lo de maneira mais autônoma. Um desdobramento do processo, iniciado em julho de 2019, é a continuidade do escaneamento do Edifício Vilanova Artigas e a discussão das potencialidades e limites da articulação nuvem de pontos e Heritage Building Information Modelling (HBIM). Como consequência desse processo de colaboração, desde 2018 a FAU USP e a Unife tem convênio acadêmico internacional assinado para dupla titulação de doutorado.

\section{CARACTERÍSTICAS DOS TRABALHOS: EDIFÍCIO VILANOVA ARTIGAS}

A FAU USP foi contemplada pelo programa Keeping It Modern, da Fundação Getty, em 2015. 0 programa é voltado a pesquisas sobre obras arquitetônicas do século XX de qualidade notável, com objetivo de oferecer subsídios para elaborar planos de gestão da conservação que guiem as ações nessas obras a longo prazo e estabeleçam paradigmas para a conservação da arquitetura do período. Foram financiadas, de 2014 a 2020, pesquisas em 77 edificações ao redor do mundoii; os resultados desses projetos estão formando uma bibliotecaiii, centro de intercâmbio de referências e de experiências. 0 programa é relevante para uma conscientização mais ampla e para instituir mudanças na abordagem das obras modernas: em vez de unicamente reagir a problemas emergenciais que se apresentam, o intuito é, a partir das pesquisas e dos dados acumulados e analisados, entender as causas de degradações para prevenir, antever e melhorar o desempenho dos diversos sistemas dos edifícios. Essa alteração de abordagem exige estudos, planejamento, monitoramento constante e ações estruturadas ao longo do tempo. A Fundação Getty proporcionou, assim, meios para a etapa fundamental - a pesquisa que subsidia a elaboração dos planos de ação - e para que se comecem a divulgar e estabelecer procedimentos e protocolos experimentais para questões recorrentes na arquitetura do século XX que ainda não foram enfrentadas de modo sistemático, não apenas no Brasil, mas também em âmbito internacional. Cinco dos projetos contemplados foram centrados na implementação de planos; em razão dos estudos de excelência realizados antes de sua aplicação e do sucesso dos resultados obtidos, esses projetos podem servir de modelo para outros edifícios do século XX.

No caso do Projeto Getty para o edifício Vilanova Artigas - construído entre 1966 e 1969, com projeto de Vilanova Artigas e Carlos Cascaldi -, o intuito não foi elaborar um plano de conservação preventiva completo, em função da duração do processo e das verbas concedidas, mas realizar pesquisas para oferecer subsídios para posterior elaboração do plano. 0 estudo teve duas ênfases: controle e gestão do principal agente de degradação do edifício ao longo de sua história, a água; análise de meios para enfrentar as consequências dos reparos realizados nas empenas de concreto em 2014, que resultou numa imagem fragmentada. 0 foco recaiu no desempenho dos elementos de proteção: a cobertura (Tarefa 2) e as empenas (Tarefa 3). A pesquisa, porém, partiu da premissa de que essas questões estão relacionadas com as formas de apreensão do edifício ao longo do tempo (Tarefa 1). Assim, a Tarefa 1 ocupou-se de sistematizar e analisar material de arquivo e bibliografia, documentar transformações e formas de apropriação do espaço, organizar dados e elaborar levantamento cadastral. 0 objetivo era elaborar análises das características espaciais e propor diretrizes para uso e tratamento dos espaços, que respeitem suas características e a 
composição do organismo como um todo, entendidas como valor a ser tuteladoiv. As propostas para o Projeto Getty estão em consonância com o Plano Diretor Participativo (PDP) para os edifícios da FAU USP, processo desenvolvido entre 2010-2011 na Faculdade, que envolveu leituras técnicas e participativas dos espaços da FAU USP e foi votado em fórum paritário, em maio e junho de 2011v. O Plano não foi pensado como projeto para execução imediata, mas como uma série de princípios, diretrizes e procedimentos que deveriam ser seguidos no futuro para fazer face à manutenção dos edifícios, estabelecendo prioridades.

As empenas são elementos de proteção e essenciais na apreensão do edifício; tiveram papel central nos estudos e, para poder analisá-las de modo adequado, foi feito o escaneamento a laser. Mesmo sendo brutalistas e deixando propositalmente explícitas as marcas do processo construtivo que lhes deu origem, as empenas sempre ofereceram uma imagem unitária do edifício; isso se perdeu com as obras realizadas em 2014, em que os reparos acabaram por criar uma imagem composta por fragmentos adossados entre si, inadequada para a percepção do organismo arquitetônico e problemática do ponto de vista técnico. Os estudos feitos durante o Projeto Getty apontam para a ineficiência dos reparos: há fissuras na pele e nas bordas dos reparos e, em trechos de reparos que foram reabertos para controle, a armação não está devidamente recoberta pela argamassa de reparo, o que resulta tanto em problemas de desempenho estrutural, quanto de proteção contra a corrosão.

O escaneamento a laser em três dimensões obtém uma nuvem de pontos das superfícies do edifício com precisão métrico-morfológica, constituindo a base para analisar as propriedades formais, geométricas, materiais e estruturais vi. Além do modelo digital ter acurácia geométrica, através dele é possível indagar os dados de refletância que, associados à inspeção visual e registros fotográficos, permite o aprofundamento cognitivo sobre as características da superfície e eventuais patologias, como será discutido adiante. 0 trabalho feito, em função dos objetivos do Projeto Getty e das características do edifício, deram origem a análises que integraram os resultados obtidos por escaneamento a laser, levantamento topográfico - com estação total para registro acurado dos pontos de controle e para a definição de rede de enquadramento articulada - e registro fotográfico sistemático do edifício e do estado de conservação de suas superfícies. O todo foi associado a um levantamento diagnóstico abrangente das superfícies para verificação de seu estado de conservação, seguido pela fase de análise e integração com os dados de refletância do escaneamento.

Esse processo permitiu obter a geometria precisa das empenas e dos reparos realizados durante a obra de 2014, sendo essencial para sua quantificação (Figura 1), dado até então não disponível. Possibilitou, ainda, verificar que não existem deformações estruturais, nem fissuras de grande porte a ponto de se tornarem visíveis no levantamento, concebido na escala 1:20, tema a ser abordado adiante. 0 escaneamento, associado a análises laboratoriais, ofereceu dados para um diagnóstico mais preciso das manifestações patológicas da superfície e de sua localização, gerando um mapeamento de um tramo da fachada que serve como base e modelo para aprofundamentos posteriores a serem feitos para todas as superfícies (Figura 2). É importante notar que não existe uma relação biunívoca entre dados de refletância e diagnóstico, que deve ser sempre construído criticamente a partir da articulação entre diversos métodos que permitam obter dados mais consistentes sobre as degradações. Mas, se para edifícios de alvenaria de tijolos e de pedra os dados de refletância e as manifestações patológicas estão mais codificados e pautados em numerosas experiências de escaneamento ao longo das últimas décadas, para os edifícios de concreto aparente isso ainda está em construção. A base utilizada foi o documento italiano Uni Normal 1/88, voltada a alvenarias de pedra e tijolos, relidas e adaptadas para os problemas do concreto aparente; a experiência nas empenas da FAU USP foi um importante passo nesse sentido, que deverá ser ainda mais bem explorado nos próximos anos. 0 escaneamento, associado a pesquisas documentais e 
iconográficas e inspeções visuais, também pode ser explorado para esclarecimentos historiográficos, como, por exemplo, no entendimento das fases de concretagem do edifício (conforme discutido na Tarefa 3).
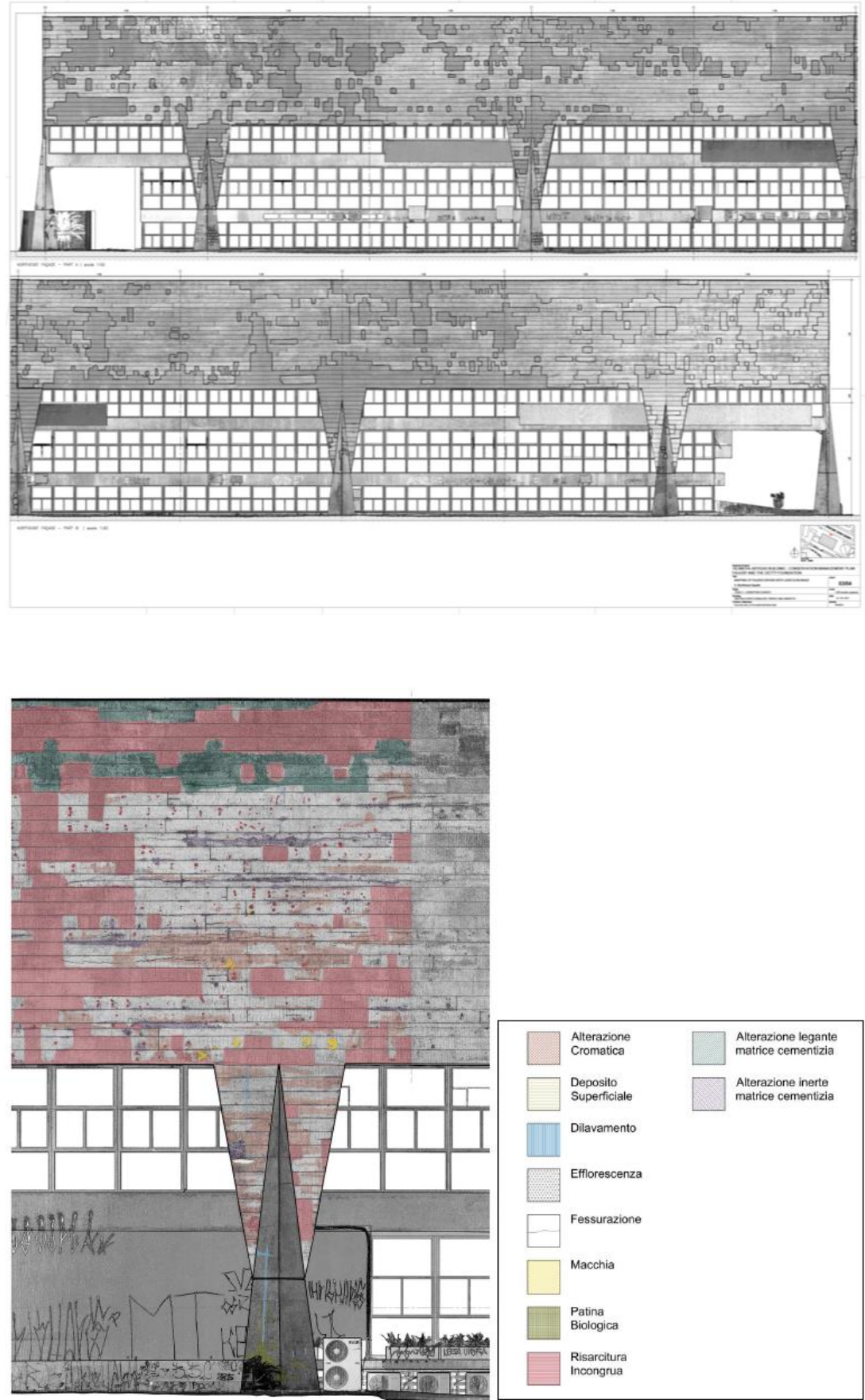

Como essa primeira fase da aproximação ao diagnóstico integrado do edifício Vilanova Artigas foi limitada às empenasvii, em função dos objetivos do projeto, a colaboração entre a FAU USP e o DIAPReM se desdobrou na continuidade do escaneamento para todo o edifício, a ser executada em etapas. A primeira delas, voltada à face externa da cobertura, foi realizada
Figura 1. Ortofoto obtida pela nuvem de pontos do Edifício Vilanova Artigas, com marcação dos reparos realizados na empena Nordeste. Escaneamento a laser realizado pelo DIAPReM.

Fonte: DIAPReM, 2016.

Figura 2. Mapeamento de danos sobre ortofoto de trecho representativo da empena Sudeste do Edifício Vilanova Artigas, realizado pela equipe do DIAPReM.

Fonte: DIAPReM, 2016. 
em julho-agosto de 2019. Previa-se a continuidade nas áreas internas do edifício em março de 2020, com o escaneamento executado pela equipe FAU USP, sob supervisão de Ferrara, mas os trabalhos tiveram de ser adiados em função da pandemia de SARS-CoV-2. Os trabalhos e o cronograma de execução serão reestruturados assim que a situação o permitir. Como mencionado, outra frente de trabalho é a de explorar as potencialidades da articulação entre nuvem de pontos e HBIM, tema coordenado, na FAU USP, por Norberto Corrêa da Silva Moura. Uma primeira modelagem, Revit-BIM, de abril de 2020, de um trecho do edifício, feita por Andrea Zattini do DIAPReM está sendo desenvolvida para gerar um modelo que possa integrar dados de informação de natureza diversa, como os resultados dos ensaios de caracterização do concreto obtidos após extração de amostras no âmbito do projeto Getty, o histórico de intervenções físico-espaciais realizadas ao longo das décadas e as análises do estado de conservação dos ambientes realizadas durante a elaboração do PDP, entre outros.

\section{CARACTERÍSTICAS DOS TRABALHOS: MUSEU DO IPIRANGA}

Os trabalhos no Museu do Ipirangaviii foram desenvolvidos como desdobramento desse processo de colaboração. A proposta foi mais ampla, se comparada à campanha realizada na FAU USP, pois foi voltada ao organismo em sua inteireza, permitindo obter a geometria precisa da construção e a morfologia da degradação de suas superfícies, além de dar continuidade à formação da equipe localix. 0 intuito foi também oferecer um produto que pudesse servir de base ao processo de modernização e restauro do edifício, fechado ao público em 2013 e objeto de concurso público de arquitetura em 2017. As obras de restauro e ampliação do Museu foram iniciadas em 2019 e a previsão é que estejam prontas em 2022.

A aquisição da nuvem de pontos, feita em agosto de 2017, foi realizada de modo paralelo a esse processo; o material foi tratado pelo DIAPReM em Ferrara, enviado à USP em abril de 2018, e novo ciclo de formação de estudantes e eventos abertos ao público ocorreu entre agosto e setembro de 2018. 0 projeto arquitetônico vencedor do concurso, do escritório Hereñú + Ferroni Arquitetos ${ }^{\mathrm{x}}$, começou a ser desenvolvido em 2018 e se beneficiou da nuvem de pontos de Ferrara, utilizando-a como base para extrair plantas e cortes para a elaboração do projeto executivo, desenvolvido ao longo de 2018 e concluído em 2019. Para tanto, o DIAPReM organizou seminário de formação para os arquitetos do escritório em Ferrara, de que participou também Renata Campiotto, estudante de pós-graduação da FAU USP.

0 trabalho no edifício do Museu também foi desenvolvido pela articulação de procedimentos diversos, com o escaneamento a laser para o levantamento abrangente do organismo e de detalhes do edifício e levantamento topográfico (com estação total), para a construção de uma rede poligonal que possibilita a verificação e o controle na fase de registro e também das relações entre o sistema de referência geral e de pormenores, permitindo, por exemplo, que trechos e unidades estruturais possam ser decompostas e analisadas com referência a esse sistema. Esses levantamentos foram complementados pelo exame visual e registro fotográfico sistemático do edifício e do ambiente em que está inserido, de suas características materiais e construtivas e do estado de conservação de materiais e superfícies, sem uso de métodos destrutivos. 0 resultado é uma nuvem de pontos georreferenciada (Figura 3), origem dos desenhos do projeto, que possibilita diversos níveis de aprofundamento de um organismo complexo, somente passíveis de ser obtidos a partir de uma base de dados precisa em 3D. Essa nuvem de pontos dá suporte à análise das características geométricas e das deformações das estruturas da cobertura, da angulação dos elementos verticais para verificar a segurança estática do edifício, e da caracterização material das fachadas que, associada aos dados de refletância, permite determinar o estado de conservação das superfícies. 
No que respeita à nuvem de pontos do Museu do Ipiranga, o treinamento de parte da equipe do escritório $\mathrm{H}+\mathrm{F}$ permitiu que fossem extraídos dados e informações para subsidiar a elaboração do projeto executivo de restauro e de ampliação. Além do levantamento métrico de alta precisão, que viabilizou as bases para os desenhos, a análise da nuvem de pontos também revelou características estruturais antes desconhecidas. Na realidade, a necessidade de uma visão integral do conjunto estrutural já havia sido apontada anteriormente nos relatórios de João Mateus (2013) e João Appleton (2013) - da Faculdade de Arquitetura da Universidade de Lisboa, e do Laboratório Nacional de Engenharia Civil de Lisboa, respectivamente -, realizados quando da interdição do edifício. Naquele contexto, foram assinalados indícios que somente foram confirmados através de seções elaboradas na nuvem de pontos resultante do escaneamento a laser (Figura 4), resultando na alteração da solução estrutural proposta pelo escritório vencedor do concurso: o terreno sob a escadaria frontal do edifício não mais poderia ser escavado para criar a conexão com a nova área subterrânea da ampliação do Museu, do modo como inicialmente previsto. Pela visualização da seção gerada na nuvem de pontos, verificou-se que as paredes ali existentes são autoportantes e suas fundações são responsáveis pelo descarregamento de forças de toda porção frontal do corpo central do edifício. Junto a uma reavaliação estrutural, sobretudo do subsolo do edifício, com novos ensaios e prospecções investigativas do terreno e com o que foi verificado com as obras em execução, foi elaborada uma solução alternativa para o projeto.
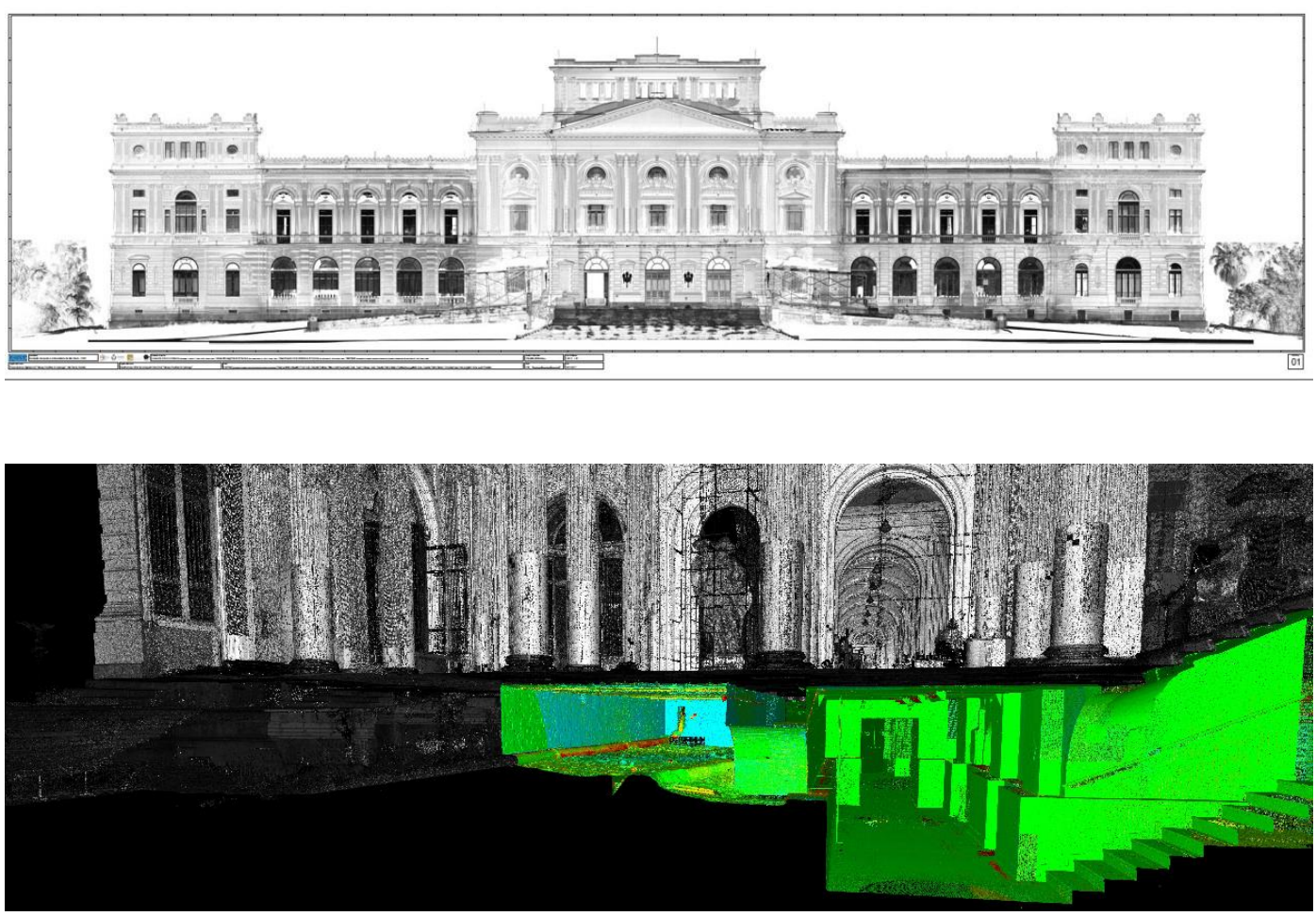

\section{SOBRE O MÉTODO E OS CICLOS DE FORMAÇÃO}

O escaneamento a laser tem sido empregado com maior frequência ao longo das últimas décadas devido à rapidez na coleta dos dados e à precisão do produto gerado. Apesar da
Figura 3. Ortofoto obtida da nuvem de pontos do Museu do Ipiranga. Escaneamento a laser $3 d$ realizado pelo DIAPReM.

Fonte: DIAPReM, 2017.

Figura 4. Perspectiva de parte seccionada da nuvem de pontos do Museu do Ipiranga, elaborada pela equipe do DIAPReM. É possível observar 0 arranjo estrutural entre as colunas do saguão e as fundações no subsolo.

Fonte: DIAPReM, 2017. 
eficácia cada vez maior do método, nem sempre sua aplicação é acompanhada de julgamento crítico, levando a conclusões precipitadas de que o equipamento laser scanner, por si só, seja capaz de realizar integralmente o trabalho de captura e processamento de dados, pulando etapas fundamentais para a correta apreensão daquilo que é o objeto de estudo e análise. Por essa razão, é necessário compreender o modo de funcionamento do aparelho, seus limites e potencialidades, que indicam a necessidade de integração com outros tipos de investigações; essa foi a tônica dos ciclos de formação na FAU USP oferecidos pela equipe do DIAPReM.

Como forma preliminar de aproximação ao método, foram explicadas as principais diferenças de operação entre cada tipo de scanner e seus alcances. A depender das dimensões e da complexidade do objeto a ser escaneado, há um tipo de aparelho mais apropriado: recomenda-se o uso de scanners que operam por triangulação, ou seja, que realizam a medição a partir da reflexão dos feixes de laser emitidos pelo scanner em um aparelho receptor, para objetos pequenos ou esculturas, uma vez que seu alcance varia entre 0,1 a 25 metros. Os scanners que operam por diferença de fase realizam a medição pela diferença das características das ondas do raio laser que são emitidas e que retornam ao aparelho, como comprimento e frequência, podendo alcançar distâncias entre 5 a 120 metros. Por fim, os scanners que operam por tempo de voo realizam a medição pelo tempo decorrido entre a emissão e o retorno dos feixes de laser, utilizando valores conhecidos de velocidade da luz e do índice de refração do meio - nesse caso, podem atingir objetos localizados a até 600 metros de distância do aparelho. Sobre esse ponto, é importante salientar que quanto maior o alcance do aparelho, maior é sua imprecisão: se o scanner por triangulação admite erros da ordem de $0,5 \mathrm{~mm}$, o scanner que opera por tempo de voo admite imprecisões de $20 \mathrm{~mm}$ (GROETELAARS, 2015). Como em toda operação de levantamento métrico, os possíveis erros devem ser levados em consideração - tanto os gerados pelo próprio instrumento, quanto os cometidos pelo operador durante a execução. Com isso, determina-se o erro máximo admissível de acordo com os objetivos do levantamento e, então, escolhe-se o método mais adequado. Como frisado por Guido Galvani, coordenador do escaneamento das fachadas da FAU USP, deve-se sempre ter em mente que "a medida exata não existe".

Uma forma de minimizar erros de execução de um levantamento métrico arquitetônico ou escaneamento a laser é realizar o planejamento pormenorizado de todas as suas etapas, antevendo possíveis interferências que poderão ocasionar incongruências métricas ou falhas durante o processamento da nuvem de pontos. A depender do objetivo do levantamento, que definirá o nível de detalhamento do produto, ou seja, a distância mínima pretendida entre os pontos da superfície do objeto a serem capturados pelo scanner, devem ser realizados testes para verificação do tempo de operação de cada varredura. Isto permitirá estimar a duração total do escaneamento e, caso ultrapasse o período previsto no cronograma de trabalho, podem ser feitos ajustes em relação à densidade da nuvem de pontos ou à quantidade de posições de locação do aparelho, bem como dos alvos ${ }^{\mathrm{xi}}$, caso sejam utilizados.

No escaneamento da face externa da cobertura da FAU USP, realizado entre julho e agosto de 2019, a importância do planejamento tornou-se evidente: dada a complexidade geométrica da estrutura de vigas invertidas, foi essencial ponderar qual seria a melhor alternativa para posicionar o scanner. A princípio, a solução que se mostrava ideal, por requerer menor número de varreduras e proporcionar menor quantidade de superfícies em sombreamento, era sobre o cruzamento das vigas. No entanto, antes mesmo de testá-la, mostrou-se inadequada, pois não permitia a correta estabilidade do aparelho, podendo comprometer os resultados gerados. Por fim, a resposta mais apropriada foi a de realizar cada escaneamento a partir da área central de módulos intercalados, viabilizando o alcance máximo da varredura e otimizando, tanto quanto possível, a quantidade de deslocamentos e o tempo da operação. 
Os dados obtidos pelo scanner são gerados de forma semiautomática, pois dependem dos parâmetros definidos pelo operador antes do início da varredura. 0 conjunto de pontos coletados, que posteriormente darão origem à "nuvem de pontos", são, na realidade, coordenadas da superfície do objeto que foram atingidas pelo feixe de raio laser do aparelho e que estão localizadas num determinado plano de referência criado pelo scanner (WEINMANN, 2016). Uma das vantagens do método é o dado qualitativo que cada um desses pontos geolocalizados pode oferecer: seu índice de refletância. Este índice representa a porcentagem de determinada propriedade física da onda de raio laser que, ao retornar ao aparelho, pode variar devido a uma série de fatores: tipo de material, temperatura, umidade, textura, angulação da superfície. Assim, dois pontos próximos que apresentem qualquer tipo de característica física diferente terão, além de suas coordenadas, valores de refletância distintos, em maior ou menor grau.

No software de processamento da nuvem de pontos, a visualização pode ser feita através de escala cromática que leva em consideração os valores de refletância, gerando um modelo em "falsa cor"xii. Com isso, é possível observar as diferenças materiais e tipológicas das superfícies, individualizando elementos distintos com base nos dados obtidos, e a frequência com que isso ocorre em determinadas áreas da edificação, através de uma observação analítica (PUCHE et al., 2017). Quando integrados a outros levantamentos e diagnósticos, esses dados podem ser auxiliares na elaboração de um mapeamento de danos ${ }^{\text {xiii, }}$, por exemplo.

No caso das fachadas do edifício Vilanova Artigas, a diferença entre o índice de refletância dos diversos pontos escaneados foi providencial para a identificação da área de reparos em argamassa nas empenas de concreto. Através da manipulação do parâmetro de cores aplicado a cada porcentagem de refletância, foi possível tornar mais ou menos evidente os perímetros de cada reparo, possibilitando seu desenho e o posterior cálculo que quantifica a intervenção realizada (Figura 5). Contudo, devido a interferências de folhagens das árvores no entorno do edifício, ou a valores de refletância muito próximos entre os trechos do concreto e da argamassa de reparo, o mapeamento só pôde ser totalmente concluído quando integrado a inspeções visuais e ao levantamento fotográfico. Essa limitação do método foi prevista no planejamento da operação: é por isso também que a avaliação do operador durante o trabalho de campo é essencial, pois além da verificação da qualidade de cada varredura, os apontamentos e análises preliminares auxiliam nas fases subsequentes, tanto no processamento e junção das várias nuvens de pontos geradas, quanto nos produtos elaborados a partir delas, que, por vezes, necessitam de complementação. Não existe fórmula que crie de forma automática a operação de escaneamento de um bem cultural: quando se trata de documentação voltada ao patrimônio, o procedimento correto envolve planejamento e integração entre diferentes técnicas ${ }^{\text {xiv, }}$, devendo-se sempre considerar a singularidade daquele bem e o local onde está inserido (LERMA et al. 2011).

Os treinamentos realizados durante os ciclos de formação na FAU USP (tratando de ambas as campanhas de escaneamento) não contemplaram a fase de processamento, em que os dados brutos capturados pelo equipamento são otimizados, resultando na nuvem de pontos limpa e pronta para a exportação de produtos bi e tridimensionais. Nessa etapa, a remoção de dados supérfluos, como vegetação e construções no entorno do edifício, interferência de transeuntes ou mobiliário é feita pelo operador de acordo com os objetivos do levantamento. A análise da precisão da nuvem de pontos pode então ser feita pela checagem manual de dimensões conhecidas ou por softwares que são capazes de calcular desvios de modelagem. 
Figura 5. Visualização da nuvem de pontos de um mesmo trecho da empena Sudeste do Edifício Vilanova Artigas com diferentes intensidades aplicadas aos parâmetros de refletância. Realizado pela equipe do DIAPReM.

Fonte: Extraído de BALZANI; MAIETTI; KÜHL, 2017.
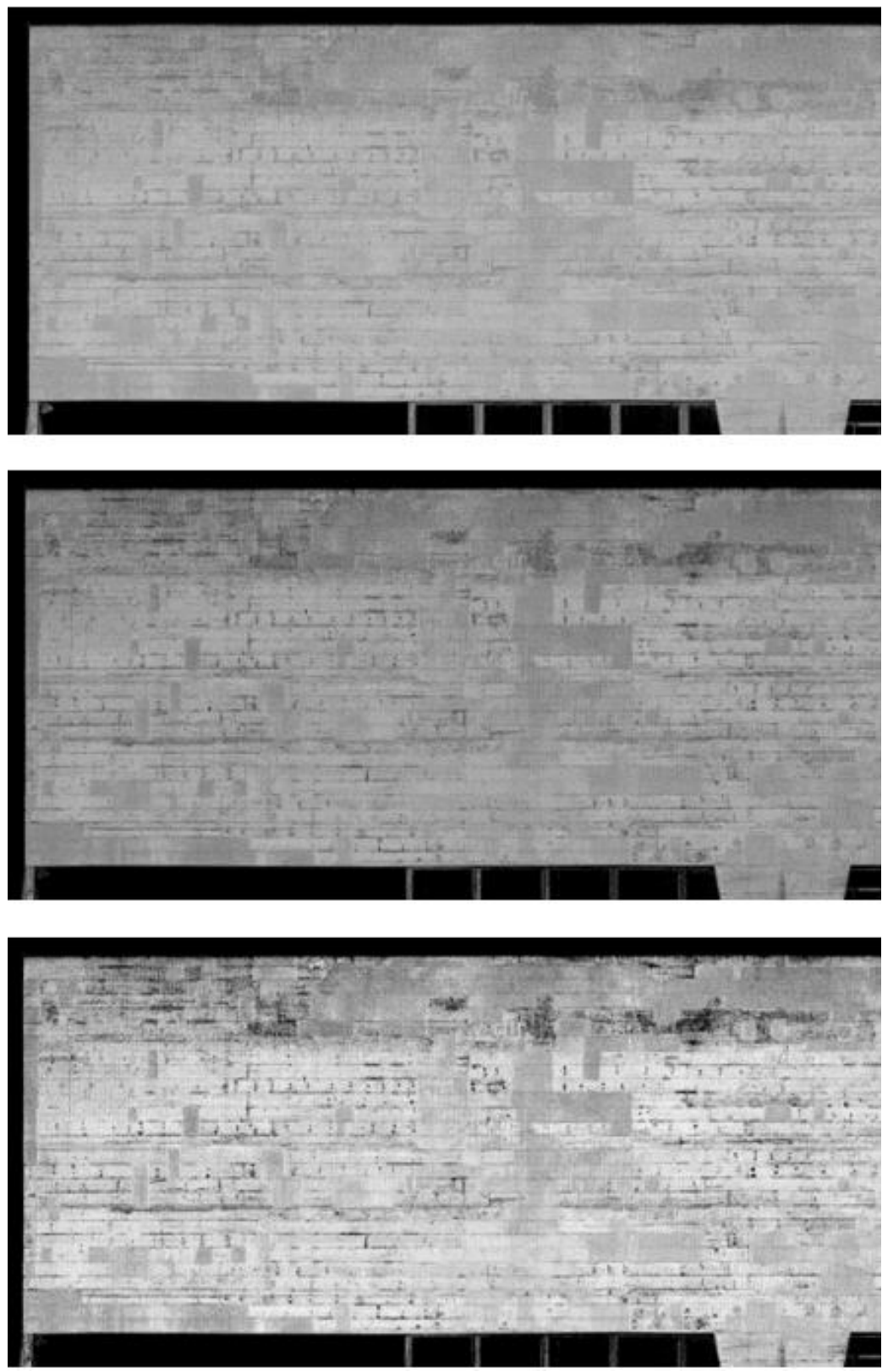

Com os arquivos resultantes dos escaneamentos dos edifícios Vilanova Artigas e do Museu do Ipiranga foram oferecidos os treinamentos do software Leica Cyclone para os alunos da FAU USP, que ocorreram em 2016 e em 2018, respectivamente. Nos ciclos de formação, além da operabilidade de navegação no programa e da extração de ortofotos, foi abordada a compreensão dos dados de bilhões de coordenadas georreferenciadas, e o uso do escaneamento como ferramenta de análise crítica. Ao manipular os parâmetros das diferentes escalas de cor que permitem a visualização da nuvem de pontos pela diferença dos índices de refletância de cada um dos pontos captados, é possível acentuar ou atenuar alterações nas superfícies escaneadas, fornecendo suporte para múltiplas análises (Figura 6). 

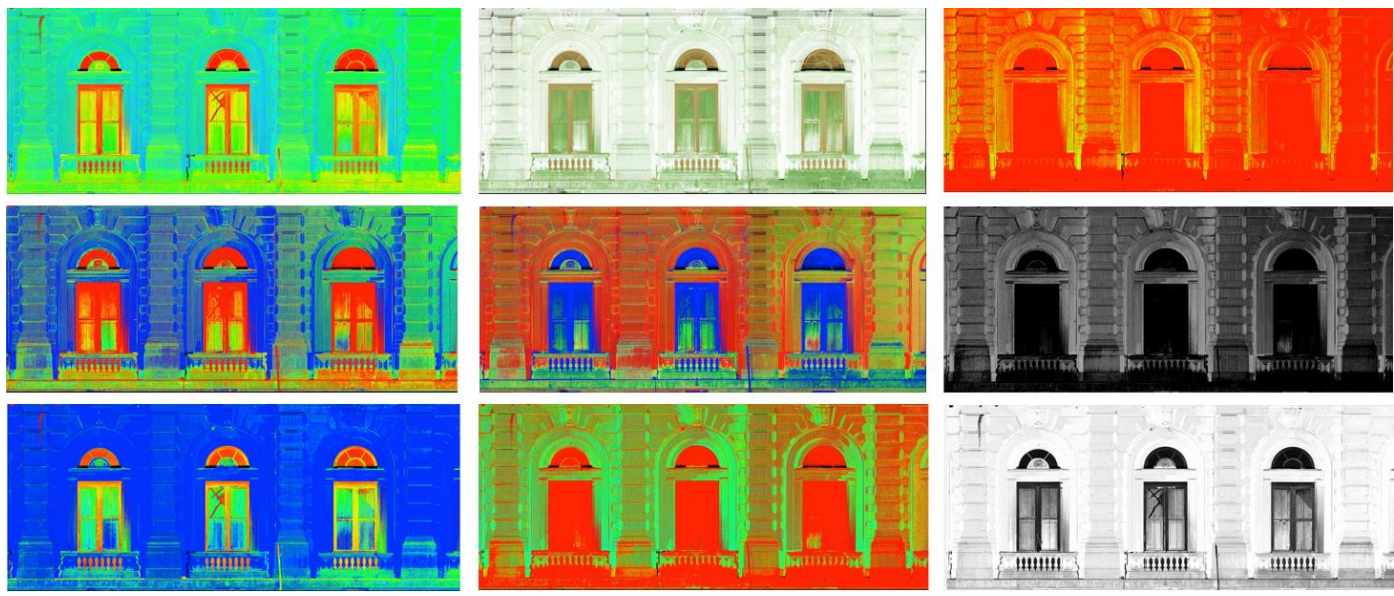

Como visto, o escaneamento, por si só e sem acompanhamento criterioso que tem início na própria escolha do método, não é capaz de fornecer resultados confiáveis. Por gerar um produto visualmente íntegro, pode dar a impressão de extrema precisão do resultado, mesmo quando feito sem critérios rigorosos de acuidade. A ideia errônea de que o scanner é capaz de realizar a varredura automaticamente e fornecer uma nuvem de pontos precisa é recorrente e muitas vezes vem acompanhada da intenção de produzir modelos HBIM de forma automatizada. É importante frisar que, por mais automático que aparente ser, o procedimento deve sempre ser relido de modo crítico em relação ao planejamento, controle e análise em todas as suas etapas, de modo que a operação não perca sua coerência e rigor ${ }^{\mathrm{xv}}$. 0 Quadro 1 contém informações sobre ambas as empreitadas, na FAU USP e no Museu do Ipiranga, evidenciando a complexidade inerente a esse tipo de levantamento.

\begin{tabular}{|c|c|c|}
\hline & $\begin{array}{c}\text { Edifício Vilanova Artigas } \\
\text { (FAU USP): Empenas }\end{array}$ & Museu do Ipiranga (MP USP) \\
\hline \multicolumn{3}{|l|}{ Dados do Escaneamento } \\
\hline Ano da realização & 2016 & $2017-2018$ \\
\hline Técnica / Instrumentos & $\begin{array}{l}\text { - Levantamento com laser } \\
\text { scanner tipo Time-Of-Flight (1 } \\
\text { Laser Scanner Leica C 10) } \\
\text { - Levantamento topográfico } \\
\text { (Leica Total Station TS11 1"R } \\
\text { 1000) } \\
\text { - Análise visual e registro } \\
\text { fotográfico sistemático }\end{array}$ & $\begin{array}{l}\text { - Levantamento com laser } \\
\text { scanner tipo Time-Of-Flight (2 } \\
\text { Laser Scanners Leica P 40) } \\
\text { - Levantamento topográfico } \\
\text { (Leica Total Station TCR 1203) } \\
\text { - Análise visual e registro } \\
\text { fotográfico sistemático }\end{array}$ \\
\hline $\begin{array}{l}\text { Dias de levantamento / horas } \\
\text { de atividade }\end{array}$ & $\begin{array}{l}15 \text { dias (incluindo Ciclo de } \\
\text { Formação) / } 1 \text { laser scanner }\end{array}$ & $\begin{array}{l}28 \text { dias totais, } 21 \text { dias úteis / } \\
208 \text { horas de uso de cada } \\
\text { laser scanner }\end{array}$ \\
\hline Número de estações & 83 estações & 1800 estações \\
\hline Coordenadas adquiridas & 6,3 bilhões & 240 bilhões \\
\hline \multicolumn{3}{|c|}{ Dados de Cooperação e Equipes } \\
\hline Parceiros & $\begin{array}{l}\text { Ferrara: DIAPReM, TekneHub } \\
\text { Technopole, Consorzio Futuro } \\
\text { in Ricerca } \\
\text { São Paulo: FAU USP, CPC USP }\end{array}$ & $\begin{array}{l}\text { Ferrara: DIAPReM, TekneHub } \\
\text { Technopole, Consorzio Futuro } \\
\text { in Ricerca } \\
\text { São Paulo: FAU USP, CPC USP, } \\
\text { Museu Paulista USP }\end{array}$ \\
\hline
\end{tabular}

Figura 6. Visualização da nuvem de pontos de um mesmo trecho da fachada Sul do Museu do Ipiranga com diferentes escalas de cor. É possível observar o realce de manchas de umidade na base dos balaústres a depender da escala de cor aplicada. Elaboração de Renata C. Campiotto, 2018.

Fonte: DIAPReM, 2018.

Quadro 1. Dados dos projetos. Elaboração dos autores a partir dos dados do DIAPReM e FAUUSP. 


\begin{tabular}{|c|c|c|}
\hline Financiamento & $\begin{array}{l}\text { The Getty Foundation, } \\
\text { Programa Keeping It Modern } \\
\text { Fundação de Apoio à } \\
\text { Universidade de São Paulo } \\
\text { (FUSP) }\end{array}$ & $\begin{array}{l}\text { Fundação de Apoio à } \\
\text { Universidade de São Paulo } \\
\text { (FUSP) }\end{array}$ \\
\hline Coordenadores científicos & $\begin{array}{l}\text { DIAPReM: Marcello Balzani } \\
\text { FAU USP: Projeto Getty } \\
\text { Coordenação Geral: Maria } \\
\text { Lucia Bressan Pinheiro; } \\
\text { Claudia T. A. Oliveira } \\
\text { Coordenação de Linhas: } \\
\text { Beatriz Mugayar Kühl; Silvio } \\
\text { Oksman (T1) } \\
\text { Antonio Carlos Barossi; } \\
\text { Rodrigo Vergili (T2) } \\
\text { Claudia T. A. Oliveira (T3) }\end{array}$ & $\begin{array}{l}\text { DIAPReM: Marcello Balzani } \\
\text { FAU USP: Beatriz Mugayar } \\
\text { Kühl }\end{array}$ \\
\hline $\begin{array}{c}\text { Coordenador do projeto de } \\
\text { levantamento }\end{array}$ & Luca Rossato (Unife) & Luca Rossato (Unife) \\
\hline $\begin{array}{l}\text { Coordenador do } \\
\text { levantamento 3D }\end{array}$ & Guido Galvani (Unife) & Guido Galvani (Unife) \\
\hline Técnico do levantamento 3D & Daniele Felice Sasso (Unife) & Daniele Felice Sasso (Unife) \\
\hline Levantamento diagnóstico & Federica Maietti (Unife) & Federica Maietti (Unife) \\
\hline Suporte técnico & Leica Geosystems Brazil & $\begin{array}{l}\text { Leica Geosystems Brazil (Rio } \\
\text { de Janeiro - RJ / São Carlos - } \\
\text { SP) }\end{array}$ \\
\hline $\begin{array}{c}\text { FAU USP: } \\
\text { Estudantes de pós-graduação } \\
\text { e de graduação que } \\
\text { participaram do ciclo de } \\
\text { formação }\end{array}$ & $\begin{array}{l}\text { Ana Paula Arato Gonçalves } \\
\text { Renata Cima Campiotto } \\
\text { Rodrigo Vergili } \\
\text { Eric M. do Amaral Palmeira } \\
\text { Letícia Ameida Chaves } \\
\text { Luiza do C. M. G. Nadalutti }\end{array}$ & $\begin{array}{l}\text { Coordenação: Renata Cima } \\
\text { Campiotto } \\
\text { Maria Vitoria Fischer Novaes } \\
\text { Stephanie Luna Galdino } \\
\text { Eduardo Ribeiro } \\
\text { Jessica Mari Hanao } \\
\text { Catherine Calognomos }\end{array}$ \\
\hline $\begin{array}{l}\text { Financiamento / organização } \\
\text { do Seminário de formação }\end{array}$ & $\begin{array}{l}\text { The Getty Foundation / FAU } \\
\text { USP }\end{array}$ & $\begin{array}{lll}\text { FUSP, FAU USP, } & \text { Museu } \\
\text { Paulista USP } & & \\
\end{array}$ \\
\hline
\end{tabular}

\section{CONSIDERAÇÕES SOBRE O ESCANEAMENTO A LASER E SEU PAPEL NO DIAGNÓSTICO INTEGRADO}

A colaboração entre a FAU USP e o DIAPReM foi construída a partir de aproximação crítica: na discussão sobre o objeto e dos objetivos da cooperação, na estruturação dos trabalhos a desenvolver, na análise crítica dos resultados obtidos e no planejamento das etapas sucessivas. Foram muito debatidas as características do escaneamento a laser e para qual finalidade seria utilizado. Os membros do DIAPReM - que trabalham na área de levantamentos com métodos avançados há mais de duas décadas ${ }^{x v i}$ - sempre foram enfáticos em relação à necessidade de aproximação crítica ao tema, pois persiste muita incompreensão em relação às questões envolvidas.

Muitos leigos, que não conhecem as características dos procedimentos, têm a ideia de que, por ser procedimento que envolve alta tecnologia, o levantamento a laser se faz sozinho, cabendo ao operador apertar alguns botões. Nada mais longe da realidade. Por outro lado, 
outros acreditam que seja apenas um método custoso e que não dá resultados adequados, percepção simetricamente inadequada. Algo explicitado na proposta de trabalho e sempre enfatizado nas apresentações públicas é que o escaneamento é método utilíssimo, mas extremamente complexo. Todas as etapas devem ser preparadas e executadas de forma rigorosa - o planejamento, o escaneamento em si, o tratamento dos dados e a análise da nuvem de pontos -, algo que exige profundo conhecimento do método, do instrumento a ser utilizado, do objeto a ser escaneado e da finalidade do escaneamento. 0 escaneamento, portanto, deve ser projetado com rigor para uma dada situação e concebido numa determinada escala.

Os trabalhos no edifício Vilanova Artigas e no Museu do Ipiranga, em função dos objetivos estabelecidos para cada um deles, foram concebidos com precisão de restauro na escala arquitetônica do organismo como um todo, na escala 1:20, que permite controlar a geometria precisa da construção, verificar a existência de deformações e de recalques, controlar o ritmo dos elementos, a relação entre cheios e vazios etc. No entanto, essa não é a escala para analisar microfissuras de revestimentos nem detalhes de caixilharia, por exemplo, que devem ser trabalhados em escala 1:5, algo que exigiria uma densidade de pontos muito maior. 0 escaneamento permite fazer esse tipo de controle, mas deve ser planejado e executado para essa finalidade específica e é importante saber se compensa - do ponto de vista de horas de escaneamento e de tratamento de dados e, portanto, também de custos - para os fins desejados. Não é por que "aparenta" ter a escala 1:1 que a nuvem tem a densidade de pontos suficiente para ser precisa nessa escala. É relevante, portanto, saber a natureza dos dados necessários para fazer o escaneamento em acordo com os objetivos buscados, caso contrário o resultado ou não terá a precisão almejada, ou terá uma quantidade colossal de dados que não serão aproveitados, havendo dispêndio de tempo com procedimentos não necessários.

O DIAPReM trabalha de maneira pautada pelo rigor em todas as etapas. A começar pelo planejamento do levantamento como um todo e do estabelecimento da precisão desejada. São rigorosos na construção da rede de referências para executar o escaneamento; por isso, trabalham de modo articulado com estação total, para estabelecer poligonal de referência com precisão, e, desse modo, ter meios de controle que evitem deformações que são causadas pela sobreposição de escaneamentos sucessivos sem rede auxiliar de verificação. Essa rede é também complementada por outra, na arquitetura do edifício, sendo escolhidos pontos-chave que são objeto de escaneamento com mais densidade, algo que permite que, em etapas sucessivas ou escaneamentos sucessivos de um mesmo edifício, existam pontos de controle que possibilitam uma sobreposição acurada. Por haver esse cuidado no estabelecimento da malha de referência, foi possível dar continuidade ao levantamento do Edifício Vilanova Artigas com rigor e não por simples aposição de duas nuvens de pontos desconexas. Os pontos estão georreferenciados e, também por isso, caso se queira dar continuidade ao escaneamento do Campus em geral e de outros edifícios, se os procedimentos forem executados com rigor semelhante, será possível integrar as diversas nuvens de pontos, cada qual concebida com uma finalidade específica, de maneira a, paulatinamente, obter a nuvem de pontos de todo o Campus.

É isso que o DIAPReM está fazendo em relação ao complexo do Parque da Independência. 0 laboratório fez, para a USP, o escaneamento do Museu e, para a prefeitura, o do Monumento à Independência. A intenção era em 2020 fazer o escaneamento do Parque e unir as diversas nuvens, constituindo uma base de dados de grande envergadura que permitiria examinar o complexo como um todo. Era também o intuito escanear fases das obras, algo impossibilitado pela pandemia, e, se a situação o permitir, realizar um escaneamento pós obras, facultando, portanto, a comparação do edifício e de seu entorno em momentos diversos. 
Além de criterioso planejamento e cuidado no estabelecimento da rede de referência, o próprio escaneamento deve ser executado com rigor, respeitando as características do aparelho utilizado, com alvos em lugares adequados que permitam a necessária sobreposição entre escaneamentos das diversas estações de maneira precisa. É necessário controlar os resultados parciais de cada escaneamento, de cada jornada, para ver se há inconsistências que demandem que o escaneamento seja refeito. Depois, os dados obtidos devem ser tratados com igual diligência, para eliminar dados redundantes e menos precisos e obter uma nuvem de fato acurada. Isso demanda muitas horas de trabalho e em todas as etapas é necessário trabalhar de modo consciencioso, o que exige profissionais gabaritados ${ }^{\mathrm{xvi}}$.

É importante ter consciência de que uma nuvem de pontos mal executada pode parecer tão acurada quanto uma nuvem bem executada. Será, porém, imprecisa e, portanto, inutilizável para fins de restauro e para qualquer trabalho que exija precisão geométrica, que só é alcançada a partir de nuvem trabalhada de modo rigoroso. Se não se souber exatamente como o levantamento foi projetado e executado e, depois, como os dados foram tratados, a nuvem poderá aparentar ser adequada, mas será imprecisa e inapropriada para análise; e, mais grave, uma nuvem mal feita pode induzir a erros de interpretação. É essencial, portanto, conhecer as características do objeto a ser levantado para projetar o levantamento, pois podem ocorrer variações significativas em função das características da obra e do grau de precisão adequado para o trabalho a ser desenvolvido. Não é necessário, por exemplo, realizar um escaneamento a laser para fazer uma operação simples de reforma em edifício de baixa complexidade; por outro lado, o método é essencial para levantar, com rigor, edifícios complexos que apresentam problemas intrincados, caso do Museu do Ipiranga e do Edifício Vilanova Artigas.

\section{CONCLUSÕES}

Como enfatizou Marcello Balzani durante os ciclos de formação, o escaneamento é primordial para conservar a memória geométrica, com precisão, de uma dada construção num determinado momento - e não a partir de retificações e dimensionamentos abstratos. Auxilia em diversas frentes de trabalho, como o próprio projeto arquitetônico e o diagnóstico estrutural, pois faculta examinar com maior acurácia desníveis e desaprumos, assim como viabiliza, por exemplo, o estudo comparativo preciso de elementos específicos. Se forem realizadas outras nuvens de pontos em momentos distintos, permite a análise comparativa pormenorizada do comportamento do edifício ao longo do tempo.

Um organismo arquitetônico é sempre complexo e, obviamente, não se limita nem pode ser reduzido às suas superfícies. No entanto, para analisar uma obra arquitetônica, contamos primordialmente com as superfícies e como elas conformam o espaço - a menos que existam partes faltantes ou desmoronadas -, sem dever utilizar métodos invasivos. As superfícies arquitetônicas trazem consigo traços e dados, que possuem, como evidencia Doglioni (2008), uma intrínseca qualidade e capacidade narrativa, que pode ser analisada de distintas maneiras ao longo do tempo, e, por isso mesmo, devem ser documentadas e preservadas numa obra de restauro, que deve preservar essa capacidade narrativa e salvaguardar a articulação dos materiais. 0 escaneamento a laser tem grande interesse também por isso, por oferecer dados mais abrangentes sobre as superfícies e por permitir uma separação metodológica entre a objetividade dos dados adquiridos da sua interpretação, consentindo que sejam indagados no futuro com diferentes olhares.

É importante ainda retomar as colocações de Balzani em diversas conferências para reiterar que sozinha a nuvem de pontos não dá respostas e que a inteligência está nos operadores e não nos aparelhos. A nuvem de pontos deve ser projetada, tratada e indagada com acuidade, 
em razão dos objetivos dos trabalhos. Conhecendo suas características e potencialidades é possível indagar o objeto e obter respostas que dificilmente seriam obtidas de outra forma; para isso é necessário saber fazer as perguntas. Além de oferecer bases geométricas com alto grau de precisão, a nuvem pode ser associada a outros procedimentos e contribuir de modo decisivo para um diagnóstico preciso que leve a projetos e procedimentos de restauração mais conscienciosos. É ainda relevante enfatizar que as experiências aqui analisadas fazem parte de um trabalho de cooperação em andamento com vistas também à transferência de tecnologia e à formação de equipes locais, já tendo obtido resultados parciais de interesse.

\section{Agradecimentos}

Os autores gostariam de agradecer à Getty Foundation e à Fundação de Apoio à Universidade de São Paulo, financiadores dos projetos desenvolvidos, assim como às instituições parceiras que possibilitaram a concretização do projeto: na Itália, o DIAPReM, TekneHub Technopole, Consorzio Futuro in Ricerca; em São Paulo, a Universidade de São Paulo e suas unidades diretamente envolvidas, a FAU USP, o CPC USP, e o Museu Paulista USP. Gostariam também de agradecer ao CNPq e à FAPESP pelas bolsas outorgadas, assim como os diversos profissionais e estudantes envolvidos nas mais variadas etapas, nomeados no Quadro 1.

\section{Referências Bibliográficas}

APPLETON, J. A. S. Museu Paulista (do Ipiranga): sobre as condições de conservação e segurança do edifício e as ações de conservação e reabilitação a empreender. Novembro de 2013. (Relatório Técnico).

BALZANI, M. et al. 3d City Modelling Toward Conservation and Management. The digital documentation of Museu do Ipiranga - USP, San Paulo, Brazil. The International Archives of the Photogrammetry, Remote Sensing and Spatial Information Sciences, v. XLIV-4/W3-2020, p. 99-106, out. 2020. DOI: https://doi.org/10.5194/isprs-archives-XLIV-4-W3-2020-99-2020.

BALZANI, M.; MAIETTI, F.; KÜHL, B. M. Point cloud analysis for conservation and enhancement of modernist architecture. The International Archives of the Photogrammetry, Remote Sensing and Spatial Information Sciences, v. XLII-2/W3, p. 71-77, mar. 2017. DOI: https://doi.org/10.5194/isprsarchives-XLII-2-W3-71-2017

BALZANI, M.; RACO, F. Integrated digital models for the representation and diagnosis in existing buildings: The Clust-ER BUILD project for the value chain innovation. In: BOLOGNESI, C. M.; SANTAGATI, C. Impact of Industry 4.0 on Architecture and Cultural Heritage, Business Science Reference, Advances in Civil and Industrial Engineering. Hershey: IGI Global, 2020, p. 181-201. DOI: http://doi:10.4018/978-1-7998-1234-0

BAROSSI, A. C. O edifício da FAU-USP de Vilanova Artigas. Coleção Obras Fundamentais. São Paulo: Editora da Cidade, 2016.

CACCIOTTI, R.; BLASKO, M.; VALACH, J. A. Diagnostic ontological model for damages to historical constructions. Journal of Cultural Heritage, v. 16 (1), p. 40-48, 2015. DOI: https://doi.org/10.1016/j.culher.2014.02.002

CONTIER, F. A. O edifício da Faculdade de Arquitetura e Urbanismo na Cidade Universitária: projeto e construção da escola de Vilanova Artigas. 2015. Tese (Doutorado em Arquitetura e Urbanismo) Instituto de Arquitetura e Urbanismo, Universidade de São Paulo, São Carlos, 2015.

DOGLIONI, F. Nel Restauro. Progetti per le architetture del passato. Venezia: Marsilio, 2008. 
ENTE NAZIONALE ITALIANO DI UNIFICAZIONE. UNI 11182: Materiali lapidei naturali ed artificiali. Descrizione della forma di alterazione. Termini e definizioni. Milano, 2006.

GEORGEPOULOS, A. Data acquisition for the geometric documentation of cultural heritage. In: Mixed reality and gamification for cultural heritage. Springer: Cham, p. 29-74, 2017. DOI: https://doi.org/10.1007/978-3-319-49607-8 2

GIANNECCHINI, A. C. Técnica e estética no concreto armado: um estudo sobre edifícios do MASP e da FAU USP. 2009. Dissertação (Mestrado em Arquitetura e Urbanismo) - Faculdade de Arquitetura e Urbanismo, Universidade de São Paulo, São Paulo, 2009.

GROETELAARS, N. J. Criação de modelos BIM a partir de "nuvens de pontos": estudo de métodos e técnicas para documentação arquitetônica. 2015. 372 p. Tese (Doutorado em Arquitetura e Urbanismo) - Faculdade de Arquitetura, Universidade Federal da Bahia, Salvador, 2015.

GRUPO FALCÃO BAUER. Diagnóstico Estrutural Completo do Edifício Monumento Museu Paulista. 15 de dezembro de 2017. (Relatório Técnico).

KÜHL, B. M.; BALZANI, M.; CAMPIOTTO, R. C. Nuvem de pontos do Museu Paulista da USP. Workshop entre o DIAPReM e a FAU-USP. Revista CPC, São Paulo, n. 27, p. 310-323, jan./jul. 2019. DOI: http://dx.doi.org/10.11606/issn.1980-4466.v14i27p310-323

LEAL, A. et al. Plano Diretor Participativo da FAU: uma proposta pactuada de intervenção nos edifícios da Escola. Pós. Revista do Programa de Pós-Graduação em Arquitetura e Urbanismo da FAU USP, v. 18(30), p. 252-269, 2011. DOI: https://doi.org/10.11606/issn.2317-2762.v18i30p252$\underline{269}$

LERMA, J. L. et al. Multispectral sensors in combination with recording tools for cultural heritage documentation, Change Over Time, v. 1, n. 2, 2011, p. 236-250. Disponível em: https://www.muse.jhu.edu/article/463083. Acesso em 22 maio 2019.

Beatriz Mugayar Kühl bmk@usp.br

Renata Cima Campiotto renata.campiotto@usp.br

Marcello Balzani bzm@unife.it

Federica Maietti mttfrc@unife.it

Luca Rossato rsslcu@unife.it

Fabiana Raco fabiana.raco@unife.it

MATEUS, J. M. Avaliação Preliminar e Recomendações para a Preservação Arquitetônica do Edifício do Museu Paulista. 10 de maio de 2013. (Relatório Técnico).

PETRELLA, Y. L. M. Museu Paulista: um edifício de técnica tradicional de construção de alvenarias. 2008. 332 p. Tese (Doutorado em Arquitetura e Urbanismo) - Faculdade de Arquitetura e Urbanismo, Universidade de São Paulo, São Paulo, 2008.

PINHEIRO, M. L. B. et al. (org.). Subsidies for a Conservation Management Plan: Vilanova Artigas Building. $\quad$ FAU USP, $2017 . \quad$ Disponível em: https://www.getty.edu/foundation/pdfs/kim/fauusp vilanova artigas building cmp2.pdf. Acesso em 22 out. 2018. (Relatório Científico).

PUCHE, J. M. et al. Automatic recognition of materials from laser scanner survey data by the reflectance method. In: International Conference on Preservation, Maintenance and Rehabilitation of Historical Buildings and Structures REHAB, 3, 2017, Braga. Proceedings.... Barcelos: Green Lines, 2017, p. 29-37.

WEINMANN, M. Reconstruction and analysis of 3D scenes. Springer: Cham, 2016.

WITTER, J. B; BARBUY, H. (Org.). Museu Paulista: um monumento no Ipiranga. História de um edifício centenário e sua recuperação. São Paulo: FIESP, 1997. 


\section{Notas}

' Como exemplo de divulgação desse processo ver KÜHL; BALZANI; CAMPIOTTO, 2019.

ii Ver dados no site do Programa:

https://www.getty.edu/foundation/initiatives/current/keeping_it_modern/index.html

iii Os relatórios estão na Keeping It Modern Report Library no site da Fundação Getty:

http://www.getty.edu/foundation/initiatives/current/keeping_it_modern/report_library/index.html

iv O relatório completo, com premissas, análises e resultados das três tarefas, além da bibliografia

de referência está disponível na biblioteca do programa Keeping It Modern. Ver PINHEIRO et al.,

2017. No que se refere à Tarefa 1, foram mobilizadas fontes documentais dos mais diversos

arquivos e vasta bibliografia, como, por exemplo, BAROSSI, 2016; CONTIER, 2015; GIANNECCHINI,

2009. Todo esse material foi essencial para o planejamento do escaneamento.

${ }^{\vee}$ Sobre o Plano ver LEAL et al., 2011. Ver ainda material disponível no site da FAU USP:

http://www.fau.usp.br/administracao/congregacao/planodiretor/arquivo.html

${ }^{v i}$ No que respeita à análise desse tema para bens culturais e para referências complementares, ver GEORGEPOULOS, 2017.

vii Para análise pormenorizada do método e dos resultados, incluindo a análise dos dados de refletância, ver BALZANI; MAIETTI; KÜHL, 2017.

viii $O$ edifício-monumento que abriga o Museu do Ipiranga foi projetado pelo arquiteto italiano radicado no Brasil, Tommaso Gaudenzio Bezzi, em 1882. Sua construção esteve ligada aos esforços de afirmação da independência brasileira, firmando-se como um grande marco celebrativo da grandeza e opulência da nação brasileira. Concluído em 1895, o edifício passou por diversas obras e intervenções ao longo do século XX, sendo a escavação do subsolo a que trouxe consequências mais severas para sua conservação, acarretando uma série de danos, extensamente relatados na bibliografia sobre o tema (PETRELLA, 2008; WITTER; BARBUY, 1997). O edifício foi interditado em 2013, para que fossem realizados diversos estudos e diagnósticos sobre a estabilidade estrutural. ix Para análise pormenorizada de questões de método e aspectos técnicos ver BALZANI et al., 2020. × Para dados do projeto, ver o site do escritório: https://www.hf.arq.br/projeto/museu-paulista/

${ }^{x i}$ Os alvos, ou targets, são pontos de controle materializados por alvos fixos colocados em pontos estratégicos das superfícies do objeto a ser escaneado. Auxiliam no processamento da nuvem de pontos, pois permitem correspondência acurada entre os produtos de cada varredura. Seu uso não é obrigatório, mas é altamente recomendado para edifícios complexos ou de grandes dimensões. xii É possível associar a cada um dos pontos capturados a cor "real" do objeto escaneado, ao se acoplar uma câmera fotográfica ao scanner, fornecendo mais informações ao levantamento. xiii Uma iniciativa neste sentido é o projeto "Clust-ER Building and Construction", coordenado pela Universidade de Ferrara. Para mais informações, ver BALZANI; RACO, 2020.

xiv As técnicas utilizadas não podem ser definidas a priori, pois dependem de uma série de escolhas ligadas a fatores como os objetivos de cada campanha, as características do objeto e a disponibilidade de meios.

v Sobre tecnologias que facilitam mas não substituem o processo ontológico para gestão e processamento de informações obtidas através de levantamentos e diagnósticos, e para bibliografia complementar sobre o tema ver CACCIOTTI; BLASKO; VALACH, 2015.

xvi Para os trabalhos realizados pelo DIAPReM ver: http://www.diaprem.unife.it/archivio-progetti

xvii Esse rigor do DIAPReM em elaborar uma nuvem de pontos apropriada ao trabalho de restauro arquitetônico, fez com que o escritório $\mathrm{H}+\mathrm{F}$ optasse por trabalhar com o arquivo produzido pela equipe de Ferrara e não com outro de menor acurácia, realizado como auxiliar ao diagnóstico estrutural. 